Reis-Góes FS, Lima FLO, Amorim, CF \& Santa Izabel, TS. (2020). Prevalence of chlamydia trachomatis infection in the brazilian female population: a review article. Research, Society and Development, 9(7): 1-16, e567974483.

\title{
Prevalência de infecção por Chlamydia trachomatis na população feminina brasileira:
}

$$
\text { um artigo de revisão }
$$

Prevalence of chlamydia trachomatis infection in the brazilian female population: a review article

\section{Prevalencia de lainfección por Chlamydia trachomatis en la población femenina brasileña: un artículo de revisión}

Recebido: 13/05/2020 | Revisado: 15/05/2020 | Aceito: 18/05/2020 | Publicado: 30/05/2020

Fabiane da Silva Reis-Góes

ORCID: https://orcid.org/0000-0002-1283-5211

Unidade de Ensino Superior de Feira de Santana, Brasil

E-mail: fabiane.sreisgoesbiomed@gmail.com

Felicson Leonardo Oliveira Lima

ORCID: https://orcid.org/0000-0001-5256-6768

Faculdade Nobre de Feira de Santana, Brasil

E-mail: felicsonleonardo@hotmail.com

Carolina Ferreira Amorim

ORCID: https://orcid.org/0000-0003-1538-6762

Unidade de Ensino Superior de Feira de Santana, Brasil

E-mail: carolina.ferreira.biomedicina@gmail.com

Tasciano dos Santos Santa Izabel

ORCID: https://orcid.org/0000-0003-0631-5217

Unidade de Ensino Superior de Feira de Santana, Brasil

E-mail: tazuefsbot@gmail.com

\section{Resumo}

Chlamydia trachomatis (CT) é uma bactéria Gram negativa que parasita células eucarióticas, com maior frequência à cérvice feminina ocasionando diversas consequências a saúde da mulher. Apresenta duas formas, a primeira denominada de corpos elementares (CE) sendo está a forma infectante e resistente que sobrevive no meio extracelular e a segunda, intitulada de corpos reticulares (CR), forma proliferativa da bactéria. A CT é um patógeno muito 
virulento, com mais de vinte sorotipos que causam patologias graves nos seres humanos. Foi realizada uma revisão de literatura a partir de estudos de artigos publicados entre os anos de 2009 a 2018, nos idiomas Português, Inglês e língua Espanhola, sendo selecionado um total de 41 artigos. O presente trabalho buscou estimar a prevalência de Chlamydia trachomatis na população brasileira, destacando a epidemiologia, características da bactéria, importância do seu diagnóstico e tratamento. No Brasil, o diagnóstico e acompanhamento das infecções por esse microrganismo ainda não é uma realidade e há poucos estudos vinculados à temática, demonstram alta prevalência da infecção. Diante disso, a falta de acompanhamento e diagnóstico desse patógeno poderá culminar em complicações futuras, visto que o resultado da infecção em longo prazo e a promoção de infertilidade são relatados em vários estudos. As pacientes do sexo feminino são as mais afetadas por esse patógeno, ainda assim, complicações podem ser relatadas no sexo masculino.

Palavras-chave: Chlamydia trachomatis; Doenças sexualmente transmissíveis; Infertilidade feminina; Papilomavirus humano.

\begin{abstract}
Chlamydia trachomatis (CT) is a Gram negative bacterium that parasitizes eukaryotic cells, most frequently in the female cervix, causing several consequences to the health of women. It presents two forms, the first called elementary bodies (EC) being the infectious and resistant form that survives in the extracellular environment and the second, called reticular bodies (CR), the proliferative form of the bacterium. CT is a very virulent pathogen, with more than twenty serotypes that cause serious pathologies in humans. The present study sought to estimate the prevalence of Chlamydia trachomatis in the Brazilian population, highlighting the epidemiology, characteristics of the bacterium, the importance of its diagnosis and treatment A literature review was carried out based on studies of articles published between 2009 and 2018, in Portuguese, English and Spanish, with a total of 41 articles selected. In Brazil, the diagnosis and monitoring of infections by this microorganism is not yet a reality and there are few studies related to the theme, showing a high prevalence of the infection. Therefore, the lack of monitoring and diagnosis of this pathogen may lead to future complications, since the long-term result of infection and the promotion of infertility is seen in several studies. Female patients are the most affected by these pathogens, yet complications can be reported in males.
\end{abstract}

Keywords: Chlamydia trachomatis; Sexually transmitted diseases; Female infertility; Human papillomavirus. 


\section{Resumen}

Chlamydia trachomatis (CT) es una bacteria Gram negativa que parasita las células eucariotas, con mayor frecuencia en el cuello uterino femenino, causando varias consecuencias para la salud de las mujeres. Presenta dos formas, la primera llamada cuerpos elementales (EC) es la forma infecciosa y resistente que sobrevive en el ambiente extracelular y la segunda, llamada cuerpos reticulares (CR), la forma proliferativa de la bacteria. La CT es un patógeno muy virulento, con más de veinte serotipos que causan patologías graves en humanos. El presente estudio buscó estimar la prevalencia de Chlamydia trachomatis en la población brasileña, destacando la epidemiología, las características de la bacteria, la importancia de su diagnóstico y tratamiento. Se realizó una revisión de la literatura basada en estudios de artículos publicados entre 2009 y 2018, en portugués, inglés y español, con un total de 41 artículos seleccionados. En Brasil, el diagnóstico y monitoreo de infecciones por este microorganismo aún no es una realidad y existen pocos estudios relacionados con el tema, que muestren una alta prevalencia de la infección. Por lo tanto, la falta de monitoreo y diagnóstico de este patógeno puede conducir a complicaciones futuras, ya que el resultado a largo plazo de la infección y la promoción de la infertilidad se observa en varios estudios. Los pacientes femeninos son los más afectados por estos patógenos, sin embargo, se pueden informar complicaciones en los hombres.

Palabras clave: Chlamydia trachomatis; Enfermedades sexualmente transmisibles; Infertilidad femenina; Virus del papiloma humano.

\section{Introdução}

Chlamydia trachomatis (CT) é uma bactéria que parasita frequentemente a cérvice feminina provocando inúmeras consequências para saúde da mulher. A CT é o principal patógeno causador de infecções urogenitais em mulheres na idade reprodutiva, observa-se que de 10 a $15 \%$ de pessoas desse grupo já tiveram contato com esse agente infeccioso no mínimo uma vez, causando doença inflamatória pélvica (DIP) (Fernandes et al., 2009; Borges et al., 2011). É classificada como uma bactéria Gram negativa pelas características de sua parede celular como a presença de lipopolissacarídeos (LPS). A CT possui duas formas: a primeira é a corpos elementares (CE) sendo a forma infectante e resistente que sobrevive no meio extracelular; a segunda, corpos reticulares (CR), forma proliferativa da bactéria (Taylor et al., 2012; Gomez, 2016). 
O gênero Chlamydia pertence à ordem Chlamydiales e a família Chlamydiaceae, sendo uma bactéria aeróbica, incapaz de promover a síntese de trifosfato de adenosina (ATP), molécula indispensável para o seu metabolismo energético (Pereira, 2016). A CT é uma bactéria patogênica, imóvel com ciclo bifásico e proliferação dentro de vacúolos citoplasmáticos da célula hospedeira (Choroszy-Król et al., 2012).

Existem quatro espécies de Clamídia: $C$. trachomatis (parasita do sistema urogenital feminino e sistema uretral masculino), a C.pneumoniae que parasita exclusivamente o pulmão dos seres humanos, a $C$. pisttaci parasita das vias áreas de alguns mamíferos e aves e por fim, a C. pecorum que parasita ovinos, caprinos e o gado (Pereira, 2016).

A espécie $C$. tracomatis é a mais virulenta com mais de vinte sorotipos que causam patologias graves nos seres humanos, está subdividida em três grandes grupos: L1, L2, L2a, L2b e L3 que acometem ao linfoma venero; A, B, Ba e C acarretando o tracoma e por fim, D, Da, E, F, G, H, I, Ia, J, Ja, K esses sorotipos levam ao desenvolvimento de uretrites, cervicites, salpingites conjuntivite e pneumonia em recém nascidos (Borgeset al., 2017).

No Brasil não há um acompanhamento efetivo do Ministério da Saúde, pois não é considerada uma doença de notificação compulsória, nem tão pouco são solicitados exames a pacientes assintomáticas (Pizzetta et al., 2011).

Em pacientes com sintomatologia aparente o exame ginecológico (Papa Nicolau) e PCR (reação em cadeia da polimerase) são solicitados, entretanto, o grande problema está nas pacientes assintomáticas que permanecem com o agente etiológico em estado crônico, acarretando diversas consequências como: esterilidade e susceptibilidade de doenças graves como HPV, continuando a transmitir o patógeno para outras pessoas (Borges et al., 2011; Luppi et al., 2011; Proto et al., 2011).

A Chlamydia trachomatis utiliza-se das vias metabólicas do seu hospedeiro, a fim de gerar energia, com objetivo de realizar seu clico de reprodução. Dessa forma, em virtude da sua incapacidade metabólica de sintetizar energia a partir da molécula de ATP, essa bactéria carece de uma fonte externa de energia para concluir seu ciclo reprodutivo intrínseco no vacúolo citosólico (Choroszy-król et al., 2012).

Por outro lado, pacientes que adquirem infecção por CT, posteriormente ficam susceptíveis a infecções de Papilomavírus humano (HPV) e outros microrganismos, desta forma, a CT deixa o sistema imunológico do miométrio menos competente, promovendo novas infecções por outros microrganismos como o HPV a acometer essas pacientes (Brandão \& Lacerda, 2010; Schust et al., 2015; Wohlmeister et al., 2016). 
O presente trabalho objetiva estimar a prevalência de Chlamydia trachomatis na população brasileira, destacando a epidemiologia, características da bactéria, a importância do seu diagnóstico e tratamento.

\section{Metodologia}

As pesquisas visam encontrar novos conhecimentos como preconiza Pereira et al. (2018). Neste trabalho, foi realizada uma revisão de literatura por meio de artigos indexados nas bases de dados: Biblioteca de Saúde Virtual; Science Direct; Lilacs; SciELO, publicados no período de 2009 a 2018, nas línguas Portuguesa, Inglesa e Espanhola. Os descritores utilizados foram: Chlamydia trachomatis; doenças sexualmente transmissíveis; infertilidade feminina; Papilomavírus humano.

Assim, foi encontrado um total de 630 materiais para consulta, sendo eles artigos, dissertações e teses, onde foram selecionados 41. Os critérios de exclusão correlacionaram-se com os materiais encontrados fora do recorte temporal ou descritos em outros idiomas, também foram excluídos artigos com informações repetidas. Para a inclusão, foram selecionados, artigos publicados no período estipulado, apresentando a prevalência da infecção em estudos na população brasileira.

\section{Resultados e Discussão}

A infecção por Chlamydia trachomatis é um problema de saúde pública de dimensão global. Todavia, essa realidade é mais acentuada em países em desenvolvimento, porém em países como os Estados Unidos da América apesar de ter um acompanhamento ginecológico para identificação da CT ainda existe uma alta prevalência da bactéria nas mulheres Americanas, revelando a gravidade do problema (Lopes, 2014; López-Corbeto et al., 2016).

Neste sentido, a DIP por CT deixa a cérvice feminina susceptível a doenças graves como a infecção pelo Vírus da imunodeficiência humana (HIV) e pelo Papilomavírus humano (HPV), isso ocorre devido à depreciação do sistema imunológico acometido pela CT nas mulheres contaminadas (CDC, 2016; Gomez, 2016).

No Brasil, essa realidade é muito grave, devido à ausência de programas do governo para realizar acompanhamento e diagnóstico ginecológico na população feminina para Chlamydia trachomatis em consultas de rotina (Robial, 2015). 
Os poucos estudos publicados no Brasil revelam uma alta prevalência CT na população brasileira, sendo escassos e obsoletos os dados epidemiológicos publicados pelo Ministério da Saúde do Brasil (Robial, 2015; Pereira, 2016). Dessa forma, é difícil traçar o perfil sociodemográfico dos grupos acometidos por essa patologia. O Papanicolau ainda é o melhor diagnóstico, entretanto, muitas brasileiras tem dificuldades de acesso a esse tipo de serviço por diversos fatores. Além disso, a população masculina, também acometida por CT ficam desassistidos pelo Papanicolau (Pereira, 2016).

A mucosa vaginal é protegida por Lactobacillus spp, esses microrganismos produzem o peróxido de hidrogênio (H2O2). Assim, o peróxido de hidrogênio tem como função acidificar o pH vaginal < 4,5, dessa forma, microrganismos patogênicos são impedidos de crescerem na mucosa, essa relação simbiótica protege a cérvice feminina dessas infecções (Pereira, 2016). Porém, devido a alguns fatores como imunidade suprimida, comportamentos de riscos e higiene inadequada, ocorre o desequilíbrio da microbiota vaginal. Assim sendo, a ausência ou diminuição da população de Lactobacillus spp é um grande fator de risco, podendo resultar em infecções por micróbios como a CT (López-Castro et al., 2012; Sessa et al., 2017).

A CT é um fator potencializador da virulência do papiloma vírus humano (HPV), mulheres que desenvolvem infecções por CT ficam susceptíveis ao HPV e consequentemente o avanço rápido dessa patologia (Wohlmeister et al., 2015). Em gestantes pode provocar aborto espontâneo, nascimentos prematuros, imaturidade pulmonar do feto, aminorrex (rompimento das membranas) no momento do parto, dessa forma o feto é contaminado no canal do parto (Gomez, 2016).

Segundo a OMS (2012), em um levantamento realizado do quadro epidemiológico global das doenças sexualmente transmissíveis (DST’s) curáveis, em adultos na faixa etária de 15 a 45 anos o número foi de 498,9 milhões de pessoas acometidas por uma DST, destes, 105,7 milhões foram identificados como portadores da CT.

No âmbito brasileiro as infecções por $C$. trachomatis não fazem parte das doenças de notificação compulsória, também não possuem uma triagem obrigatória para mulheres em suas consultas ginecológicas. Além disso, muitas portadoras são assintomáticas, o que agrava ainda mais o quadro, tendo um grande impacto na saúde reprodutiva da mulher. Na população brasileira sexualmente ativa, são mais de 92 milhões de pessoas acometida pela CT, com prevalência maior em jovens, sendo a maior causa de morbidades sexuais desse grupo, pois um indivíduo assintomático não tratado atua como reservatório da CT e infectam seus parceiros (Fernandes et al., 2014). 
Borges et al. (2011), realizaram um estudo em Jundiaí em São Paulo onde foram analisados 28 pacientes do sexo feminino com idade entre 17 a 83 anos e apontaram que a prevalência da CT no grupo estudado, em 10,7\% dos casos, foram positivos para a Chlamydia trachomatis, 21,4\% das amostras demonstraram alterações em sua citologia e 7,2\% dos casos apresentaram lesão no colón do útero.

Levando em consideração o tamanho da amostra do estudo, o índice de infecção por CT é muito alto e grau das infecções encontram níveis avançados a ponto de promover lesões, isso reflete o descuido em relação a saúde ginecologia e muitas vezes por não as pacientes serem assintomáticas.

Santos et al. (2017), realizaram estudos no estado do Amazônas e revelaram alta prevalência de infecções por Chlamydia trachomatis. A incidência e prevalência são maiores do que em outros estados brasileiros como São Paulo que possui um maior número de habitantes, a amostra do estudo foram 122 pacientes voluntárias, 82 pacientes com idade de 25 anos tiveram as amostras positivas em torno de $67,2 \%$, sendo a faixa etária com maior incidência. A prevalência de Chlamydia trachomatis é demonstrada na Tabela 1:

Tabela 1 - Prevalência de Chlamydia trachomatis sexualmente transmissível na população do sexo feminino entre os anos de 2009 a 2018.

\begin{tabular}{cccc}
\hline Autor/Ano & $\begin{array}{c}\mathbf{N}^{\mathbf{0}} \text { população } \\
\text { estudada }\end{array}$ & $\begin{array}{c}\mathbf{N}^{\mathbf{0}} \text { população com } \\
\text { Chlamydiatrachomatis }\end{array}$ & $\begin{array}{c}\text { (\%) de } \\
\text { infectados }\end{array}$ \\
\hline Fernandes, Daher, Nuzzi\& Petta, 2009 & 177 & 31 & 13,5 \\
\hline Brandão \& Lacerda, 2010 & 96 & 11 & 22 \\
\hline Pizzetta et al., 2011 & 335 & 36 & 10,7 \\
\hline Herkenhoff et al., 2012 & 287 & 168 & $58,54 *$ \\
\hline Garcês et al.,2013 & 200 & 22 & 11 \\
\hline Proto et al., 2014 & 120 & 48 & 24,39 \\
\hline Matos et al.,2015 & 328 & 80 & 18 \\
\hline Santos et al.,2016 & 122 & 22 & 11,9 \\
\hline Santoset al., 2017 & 454 & 54 & 12 \\
\hline Faria et al.,2018 & 100 & 12 & \\
\hline
\end{tabular}

Fonte: autoria própria (2020).

A tabela traz a realidade da prevalência de CT no Brasil entre os anos de 2009 a 2018. Assim, é possível perceber o quanto é frequente a infecção da CT caracterizando um grave problema de saúde pública. Analisando o estudo de Fernandes e colaboradores (2009) é possível observar uma porcentagem de 13,5 pessoas infectadas pela $C$. trachomatis, o que 
chega a um ponto crítico de 58,54\%, em estudo realizado por Herkenhoff et al. (2012). No ano de 2013 houve uma redução na incidência de casos, todavia em 2014 a incidência voltou a crescer, sendo expresso um valor de 40\% (Proto et al., 2014). Esses números entram em declínio e variação com o passar dos anos, regredindo para 12\% em 2018 (Faria et al., 2018). Entretanto, esses números podem não representar a realidade epidemiológica, já que é uma infecção assintomática e sem notificação compulsória. Logo, esses resultados mostram como a infecção por CT é recorrente em nosso cotidiano.

O rastreamento para o diagnóstico da Chlamydia trachomatis não é uma tarefa fácil, a maioria dos casos são assintomáticos e quando apresentam sintomas é necessária à associação entre os sinais clínicos, tais como: secreção mucopurulenta endocervical; eritema; sangramento de mucosa. Como a maiorias dos sinais clínicos são similares a outras IST's, exames laboratoriais são necessários para um diagnóstico mais preciso (Valladão et al., 2011).

As IST's vêm crescendo consideravelmente nos últimos anos, principalmente, no que se refere a mulheres em idade sexualmente ativas (Pereira, 2016). A identificação de portadores de CT ainda é um desafio no Brasil, devido, a grande quantidade de infecções assintomáticas. Atualmente, existem vários métodos para a detecção de CT em amostras de material biológico: exame direto (Giemsa), cultura de células para obter o patógeno viável, caso queira realizar pesquisas, imunofluorescência direta (IFD), imunoenzimáticos (ELISA) direto e indireto, métodos de amplificação de ácidos nucléicos (NAATs), a sonda de DNA (captura híbrida) utilizado pelo SUS para o diagnóstico da CT e por fim, o Papanicolau pode evidenciar a presença da bactéria nas células da cérvice feminina (Gomez, 2016).

Segundo Neves et al. (2016), diversos métodos moleculares podem ser utilizados para diagnóstico da CT como PCR multiplex (reação em cadeia da polimerase), para detecção do DNA da CT, sendo altamente sensível e específico.

As principais técnicas de detecção direta são: Método direto (citologia), Cultura de células, Ensaio Imunoenzimático (Elisa direto e indireto) e a Imunofluorescência direta. O método citológico, é uma técnica que era utilizada para o diagnóstico direto através da coloração de Geisma, diagnosticando, assim infecção ocular em crianças, através da visualização e pesquisa de corpúsculo de inclusão nas células afetadas. Entretanto, esta técnica está em desuso, devido ao surgimento de métodos mais sensíveis como os sorológicos e moleculares (Serras et al., 2010).

A técnica de coloração das lâminas através do exame Papanicolau da secreção da cérvice feminina coletada no momento do exame possibilita visualizar os vacúolos de 
inclusão nas células da cérvice uterina no momento do preventivo, todavia não é o método mais sensível, porém pode-se diagnosticar a infecção pela Chlamydia trachomatis (Flores et al., 2011).

Siqueira (2017), afirma que a cultura de células já foi considerada o padrão-ouro para diagnóstico, todavia, por ser uma técnica cara e demorada e por ser menos sensível e menos específicas está sendo substituída por testes moleculares e testes imunoenzimáticos. Por outro lado, a cultura celular é muito utilizada em experimentos de pesquisas para desenvolvimento de novos testes e estudo da Chlamydia trachomatis para novos fármacos e mecanismo de infecção da mesma, sendo muito importante para novas descobertas.

Flores et al. (2011), ressalta que a CT tem tropismo pelas células McCoy e Hela, estas células crescem em monocamada. Para confirmar o diagnóstico analisa-se a inclusão no citosol celular de CRs e CEs, sendo necessário realizar uma no tecido em cultivo através de uma coloração com anticorpos monoclonais florescentes, sendo mais específico que o método de Geisma (Gomez, 2016).

O Ensaio Imunoenzimático do tipo Elisa direto, consiste em um para detecção de antígeno da Chlamydia trachomatis, sendo a proteína LPS presentes na membrana bacteriana, sendo uma técnica menos custosa e mais rápida quando comparada ao cultivo celular (Siqueira, 2017). Entretanto, pode haver reações cruzadas, pelo emprego do anticorpo antiLPS, pois muitas bactérias Gram negativas possuem este tipo de proteína gerando ligações cruzadas com outras bactérias da flora promovendo resultados falsos positivos, diminuindo assim, sua especificidade (Ferreira, 2010). No teste de Elisa são investigadas as duas classes de imunoglobulinas mais abundantes do plasma, são elas: IgG sendo o anticorpo de fase crônica que indicando que esta paciente já esteve em contato com a CT e o IgM sendo um anticorpo de fase aguda, indicado que a paciente está sendo acometida pela CT naquele momento (Siqueira, 2017).

Segundo Ferreira (2010) e Santos et al. (2017), a Imunofluorescência direta corrobora para a pesquisa direta de antígeno proveniente da Chlamydia trachomatis, onde o material biológico coletado da cérvice feminina é fixado em uma lâmina de vidro, o princípio deste teste é que se houver presença da bactéria ocorrerá uma imunorreação entre o antígeno MOMP presente na membrana da bactéria e anticorpo monoclonal anti-MOMP acoplado a uma molécula fluorocrômica. De acordo com Serras, (2010) após a formação do imunocomplexo, uma florescência será emitida na cor verde, sendo possível ser visualizada com o auxílio de um microscópio fluorescente. 
O Ensaio Imunoenzimático (Elisa indireto) é um teste com o custo relativamente baixo em relação a outras técnicas podendo ser usado para diagnóstico da C. trachomatis, sendo um método qualitativo e semiquantitativa das imunoglobulinas, IgG e IgM, sendo realizado em microplacas, tubos ou pérola (Flores, et al., 2011; Matos et al., 2015).

Assim, neste teste indireto contém anticorpos monoclonais anti-Chlamydia fixados em pérolas plásticas ou poços de placas, está fase é chamada de parte solida da reação, após a amostra ser diluído é adicionada ao poço, caso haja presença de LPS (antígeno) na amostra , as mesma se ligaram imediatamente aos anticorpo anti-Chlamydia presentes nos poços, essa amostra deve ser acondicionada na temperatura ideal para que ocorra está reação. Sendo, necessário uma lavagem para retirar ligações inespecíficas e em seguida um anticorpo IgG anti-Chlamydia produzido em coelho é adicionado a reação. Logo, na próxima etapa ocorrerá outra lavagem e um anticorpo anti-IgG de coelho com uma enzima é adicionado na reação, a amostra é encubada em temperatura e tempo adequado de acordo com o fabricante e em seguida ocorre mais uma lavagem na última fase é adicionada a reação um substrato para que a peroxidase quebre o mesmo e promove a reação cor, além do revelador da reação OPD (ortofenilenodiamina) (Almeida, 2014; Flores, et al., 2011).

Os dados obtidos demonstram o quão deficiente são as informações acerca da prevalência de CT na população feminina brasileira corroborando com a ideia de Robial, (2015) e Pereira (2016), da necessidade de um apanhado epidemiológico da IST por Chlamydia trachomatis. Logo, o que se tem na literatura sobre infecções de CT consiste em pesquisas isoladas realizadas por alguns grupos de pesquisadores, pois o Ministério da Saúde não considera a doença de notificação compulsória, o que dificulta o registro de casos na população feminina brasileira, cujo os poucos estudos demonstraram sua alta prevalência, como demostrado na Tabela 1.

\section{Considerações Finais}

Diante do exposto, foi demonstrada a realidade da população brasileira e mundial em relação à prevalência da Chlamydia trachomatis, o quão é recorrente e negligenciada, principalmente no Brasil, sendo um grave problema de saúde pública no mundo todo, principalmente em países em desenvolvimento. Assim sendo, a infecção por Chlamydia trachomatis é uma das doenças inflamatórias pélvicas (DIP's) mais graves que acometem o sistema reprodutor feminino com quadro inflamatório, tornando mulheres em idade 
(CC BY 4.0) | ISSN 2525-3409 | DOI: http://dx.doi.org/10.33448/rsd-v9i7.4483

reprodutiva inférteis, porém a CT também acomete homens e os mesmos são transmissores da bactéria, apesar de existir poucos estudos com homens.

Atualmente, possuem diversos métodos diagnósticos para a CT, porém não é simples, por não ser identificada precocemente na grande maioria dos casos, o que dificulta o tratamento. Ademais, outros testes como Técnicas de biologia molecular (reação em cadeia da polimerase - PCR) e Histopatologia são altamente precisos e podem contribuir para tratamentos e prognósticos mais satisfatórios.

Embora esteja claro o potencial de patogenicidade das infecções ocasionadas pela CT, acredita-se que uma maior atenção por parte do Sistema Único de Saúde, para estes casos, venha contribuir para a diminuição de seus agravos, em especial, a esterilidade. Sendo necessários mais estudos clínicos específicos para uma melhor compreensão dos seus mecanismos patológicos com a finalidade de propor novas terapias com maior especificidade para a doença.

\section{Referências}

Almeida NCC. (2014). Marcadores da infecção por Chlamydia trachomatis, chlamydia pneumoniae e da resposta imunológica e inflamatória na formação da placa de ateroma e progressão à doença cardíaca. Doctoral dissertation, Tese Doutorado em Biologia de Agentes Infecciosos e Parasitários - Belém, Universidade Federal do Pará.

Brasil. (2015). Protocolo clínico e diretrizes terapêuticas infecções sexualmente transmissíveis. Min. Saúde, \& Comissão Nacional de Incorporação de Tecnologias no SUS.

Borges JBR, Marchesini AC Stefani, LFBD, Belintani MVG \& Santos TAD. (2011).

Prevalência de infecção por Chlamydia trachomatis em mulheres assistidas no ambulatório de patologia do trato genital inferior da Facul.Jundiaí, Brasil. Einstein (São Paulo), 9(3), 332-6.

Brandão CRAB \& Lacerda HR. (2010). Frequência de Papilomavírus humano (HPV) e Chlamydia trachomatis em gestantes. Epidemiol. Serv. Saúde, Brasília, 19(1)43-50.

Carvalho NS, Pehgoraro A, Takimura M, Oliveira J \& Fernando C. (2010). Prevalência da infecção por Chlamydia Trachomatis em parturientes jovens atendidas em uma maternidade pública. DST - J bras Doenças Sex Transm, 22(3), 141-44. 
Centers for Disease Control and Prevention. (2016). Sexually transmitted disease surveillance. Disponível em: https://www.cdc.gov/std/stats16/CDC_2016_STDS_Reportfor508WebSep21_2017_1644.pdf.

Choroszy-Król IC, Frej-Mądrzak M, Jama-Kmiecik A, Bober T \& Jolanta SJ. (2012). Characteristics of the Chlamydia trachomatis species-immunopathology and infections. $A d v$ Clin Exp Med, 21(6), 799-808.

Dal-Pogetto MRB, Marcelino LD, Carvalhaes MADBL, Rall VLM, Silva MGD \& Parada CMGDL. (2012). Características de população de profissionais do sexo e sua associação com presença de doença sexualmente transmissível. Revista da Escola de Enfermagem da USP, 46(4), 877-83.

Fernandes AMDS, Daher G, Nuzzi RXDP \& Petta CA. (2009). Infecção por Chlamydia trachomatis e Neisseria gonorrhoeae em mulheres atendidas em serviço de planejamento familiar. Revista Brasileira de Ginecologia e Obstetrícia, 31(5), 235-40.

Fernandes LBL, Arruda JT, Aprobato S \& Garcia-Zapata, MTA. (2014) Infecção por Chlamydia trachomatis e Neisseria gonorrhoeae: fatores associados à infertilidade em mulheres atendidas em um serviço público de reprodução humana. Rev Bras Ginecol Obstet, 36(8), 353-8.

Faria, AMV, Grassi, VMT, Telles, TM \& Silva, MSN. (2018). Detecção de Chlamydia trachomatis em amostras cervicais através da reação em cadeia da polimerase. Revista de Iniciação Científica da Ulbra, 1(16).

Fresse AJ, Sueur F \& Hamdad. (2010). Diagnosis and follow-up of genital chlamydial infection by direct methods and by detection of serum $\operatorname{IgG}, \operatorname{IgA}$ and secretory IgA. Indian Journal of Medical Microbiology, 28(4):326-31.

Ferreira GRON. (2010). Soroepidemiologia de Chlamydia trachomatis, Chlamydia pneumoniae e Treponema pallidum nas aldeias indígenas Bakajá, Apyterewa, Xingu e 
Mrotidjãm, Altamira, Pará, Brasil. Dissertação de mestrado em Agentes Infecciosos e Parasitários - Belém, Universidade Federal do Pará.

Filho, ACM, Sardinha, JFJ, Costa, EP, Silva, SS \& Martinez-Espinosa, FE. (2010). Prevalência de infecção por HIV, HTLV, VHB e de sífilis e clamídia em gestantes numa unidade de saúde terciária na Amazônia ocidental brasileira. Rev Bras Ginecol Obstet, 32(4), 176-83.

Flores BCTCP, Oliveira AV, Pires MM, Goveia VA \& Brenna SMF. (2011). Chlamydia trachomatis e infecções genitais femininas. Science in Health, 2(1), 55-63.

Garcês, AX, Martinez, AMBD, Gonçalves, CV, Germano, FN, Barral, MFM \& Vieira, VC. (2013). Prevalência de Chlamydia trachomatis e fatores de risco associados à infecção detectada em amostra endocervical. Rev. Bras.Ginecologia e Obstetrícia, 35(8), 379-83.

Gomez DB. (2016). Prevalência de Chlamydia trachomatis em mulheres inférteis e gestantes assintomáticas. Dissertação de mestrado em Ciências da Saúde: Ginecologia e Obstetrícia, Faculdade de Medicina -Porto Alegre, Universidade Federal do Rio Grande do Sul.

Haggerty CL, Gottlieb SL, Taylor BD, Low N, Xu F \& Ness RB. (2010). Risk of sequelae after Chlamydia trachomatis genital infection in women. The Journal of infectious diseases, 201(Supplement_2), S134-S155.

Herkenhoff ME, Gaulke R, Vieira LL, Ferreira PDS, Pitlovanciv AK \& Remualdo VR. (2012). Prevalência de Chlamydia trachomathis em amostras endocervicais de mulheres em São Paulo e Santa Catarina pela PCR. Jornal Brasileiro de Patologia e Medicina Laboratorial, 48(5), 323-7.

Lopes SR. (2014). Prevalência de Chlamydia trachomatis em casos de partos pretermo atendidos no Hospital Universitário Cassiano Antonio Moraes, Vitória - ES. Dissertação de mestrado em Doenças Infecciosas - Vitória do Espírito Santo, Universidade Federal do Espírito Santo. 
(CC BY 4.0) | ISSN 2525-3409 | DOI: http://dx.doi.org/10.33448/rsd-v9i7.4483

López-Castro TM, Rojas-Díaz EL, Rojas-Rojas FN, Díaz-Yamal IJ \& Muñoz-Cerón J. (2012). Mecanismos de modulación de la respuesta inmune por Chlamydia trachomatis asociados a infertilidad. Revista Colombiana de Obstetricia y Ginecología, 63(4), 346-355.

López-Corbeto, E, González, V, Bascunyana, E, Humet, V \& Casabona, J. (2016). Tendencia y determinantes de la infección genital por Chlamydia trachomatis en menores de 25 años. Cataluña 2007-2014. Enfermedades Infecciosas y Microbiología Clínica, 34(8), 499-504.

Luppi, CG, Oliveira, RLSD, Veras, MA, Lippman, SA, Jones, H, Jesus, CHD \& CaiaffaFilho, H. (2011). Early diagnosis and correlations of sexually transmitted infections among women in primary care health services. Revista Brasileira de Epidemiologia, 14(3), 467-477.

Machado, MSC, Gomes, ILC, Santana, IU \& Grassi, MFR. (2012). Prevalence of cervical Chlamydia trachomatis infection in sexually active adolescents from Salvador, Brazil. The Brazilian Journal of Infectious Diseases, 16(2), 188-191.

Matos, MPD, Machado, AP, Silva, ZBD \& Barbosa, DA. (2015). Detecção de chlamydia trachomatis através de testes imunológicos em população feminina adolescente e adulta na grande Cuiabá, Mato Grosso. J bras. doenças sex. transm., 16-21.

Michelete, LA, Dias, MMB, Silva, ACF, Cabral, CS, de Abreu, LC \& Drezett, J. (2012). Infecção por Chlamydia trachomatis e Neisseria gonorrhoeae em mulheres adolescentes e adultas vítimas de violência sexual na região metropolitana de São Paulo. Reprod. Clim., 27(2), 58-64.

Neves, D, Sabidó, M, Bôtto-Menezes, C, Benzaken, NS, Jardim, L, Ferreira, C \& Benzaken, AS. (2016). Evaluation of screening for Chlamydia trachomatis among young women in primary health care services in Manaus, Amazonas State, Brazil. Cadernos de Saúde Pública, 32, e 00101015.

Regional para a África, C. (2016). Declaração do Presidente do subcomité do programa à sexagésima sexta sessão do Comité Regional (No. AFR/RC66/3). Organização Mundial da Saúde. Escritório Regional para a África. Disponível em: https://www.afro.who.int. 
(CC BY 4.0) | ISSN 2525-3409 | DOI: http://dx.doi.org/10.33448/rsd-v9i7.4483

Pereira, JDS. (2016). Detecção de Chlamydia trachomatis em amostras endocervicais de mulheres HIV soropositivas de Palhoça/SC. Dissertação de mestrado em Farmácia - Análises Clínicas - Florianópolis, Universidade Federal de Santa Catarina.

Pereira AS et al. (2018). Metodologia da pesquisa científica. [e-book free]. Santa Maria. Ed. UAB/NTE/UFSM. Disponível em:

https://repositorio.ufsm.br/bitstream/handle/1/15824/Lic_Computacao_MetodologiaPesquisa-Cientifica.pdf? sequence $=1$.

Pizzetta, RCPS, Carvalho, NS, Andrade, RP, Pizzetta, G \& Pizzera, SRC. (2011). Prevalência da infecção por Chlamydia Trachomatis e Neisseria Gonorrhoea em mulheres jovens sexualmente ativas em uma cidade do Sul do Brasil. Rev Bras Ginecol Obstet, 33(11), 328-33.

Proto, IAC, Carneiro, JM, Passos, XS, Ataídes, FS \& Amaral, WNA. (2013). Prevalência de Chlamydia trachomatis em mulheres submetidas à fertilização assistida em Goiânia. Rev. Reprod. clim., 28(3)108-111.

Schust, JDA, Ibana, JR, Buckner, L, Ficarra, M, Sugimoto, JM, Amedee, A \& J Quayle, A. (2012). Potential mechanisms for increased HIV-1 transmission across the endocervical epithelium during C. trachomatis infection. Current HIV research, 10(3), 218-227.

Sá, MI, Silva, MT, Almeida, D, Vieira, B, Lima, T, Conde, C \& Oliveira, T. (2015). Infeções sexualmente transmissíveis e factores de risco nas adolescentes e jovens: Dados de um Centro de Atendimento a Jovens. Nascer e Crescer, 24(2), 64-69.

Santos, LM, Souza, IRA, Holanda, LHC; Vaz, JOL, Tsumi, MY, Ishikawa, EAY \& Souza, MS. (2016). Alta incidência da infecção urogenital por Chlamydia trachomatis em mulheres parturientes de Belém, Estado do Pará, Brasil. Rev. Pan-Amaz Saúde. 7(4), 101-106.

Santos, LMD, Ulian, WL, Trindade, JQ, Sousa, FDMD, Oliveira, JFG, Pereira, CCC \& Sousa, MSD. (2017). Prevalência da infecção endocervical de Chlamydia trachomatis em universitárias do estado do Pará, Região Amazônica, Brasil. Revista Pan-Amazônica de Saúde, 8(3), 27-33. 
Sessa, R, Pietro, DM, Filardo, S, Bressan, A, Mastromarino, P, Biasucci, AV\& Valenti, P. (2017). Lactobacilli-lactoferrin interplay in Chlamydia trachomatis infection. Pathogens and disease, 75(5), ftx054.

Serras, AFD. (2011). Chlamydia trachomatis: genótipos circulantes, comparação de métodos para o seu diagnóstico e necessidade do seu rastreio. Dissertação de Mestrado em Microbiologia Médica - Lisboa, Universidade Nova de Lisboa.

Siqueira CR. (2017). Infecção genital causada pela bactéria Chlamydia trachomatis e o diagnóstico laboratorial (Monografia). Faculdade Sudoeste Paulista de Itapetininga, Itapetininga.

Taylor BD, Darville T, Ferrell R.E, Kammerer C. M, Ness RB, \& Haggerty CL. (2012). Variants in toll-like receptor 1 and 4 genes are associated with Chlamydia trachomatis among women with pelvic inflammatory disease. Journal of Infectious Diseases, 205(4), 603-609.

Valladão AS, Costa CMDSP, Camargo JAD \& Soares VCG. (2011). The effects of Chlamydia trachomatis on human reproduction. Rev. do Instituto Adolfo Lutz, 70(4), 457-62.

Wohlmeister D, Vianna DRB, Helfer VE, Gimenes F, Consolaro MEL, Barcellos RB \& Pilger DA. (2016). Association of human papillomavirus and Chlamydia trachomatis with intraepithelial alterations in cervix samples. Memórias do Instituto Osw.Cruz, 111(2), 106-13.

\section{Porcentagem de contribuição de cada autor no manuscrito}

Fabiane da Silva Reis Góes - 25\%

Felicson Leonardo Oliveira Lima $-25 \%$

Carolina Ferreira Amorim - 25\%

Tasciano dos Santos Santa Izabel - 25\% 